\title{
An interatomic potential study of the properties of gallium nitride
}

\author{
Peter Zapol $\dagger$, Ravindra Pandey† and Julian D Gale $\ddagger$ \\ $\dagger$ Department of Physics, Michigan Technological University, Houghton, MI 49931, USA \\ $\ddagger$ Department of Chemistry, Imperial College of Science, Technology and Medicine, South \\ Kensington, London SW7 2AY, UK
}

Received 21 March 1997, in final form 29 August 1997

\begin{abstract}
A set of interatomic pair potentials were derived for gallium nitride within the shellmodel approach. It was shown that the potential set successfully reproduces the properties of the fourfold-coordinated wurtzite and zinc-blende structures as well as the sixfold-coordinated rocksalt structure. The high-pressure phase transition from wurtzite to rock-salt structure is correctly described yielding the phase transition pressure of $50 \mathrm{GPa}$. The calculated formation energies of intrinsic point defects reveal that vacancies are the dominant native defects in GaN. Lastly, the calculated structure relaxation of the zinc-blende (110) surface predicts a layer rotation angle of $9^{\circ}$ in agreement with the results of recent first-principles calculations.
\end{abstract}

\section{Introduction}

Gallium nitride has recently emerged as a semiconducting material with promising applications in short-wave light-emitting devices and high-temperature high-power electronics [1]. These applications usually require thin films of GaN grown by molecular beam epitaxy techniques in the hexagonal wurtzite and cubic zinc-blende phases. The lack of native substrates leads to a substantial number of defects in the material. For instance, n-type conductivity of as-grown $\mathrm{GaN}$ is attributed to intrinsic defects introduced during the MBE growth. In order to understand the mechanisms of growth of defect-free and doped GaN, extensive efforts, both theoretical and experimental, are under way to study the structural and electronic properties of its crystal phases, its surfaces, native point defects and impurities.

Atomistic calculations based on empirical interatomic potentials are now routinely performed to study intrinsic and extrinsic disorder in a wide variety of materials. Many successful applications and the modest requirements for computer resources of the method make it attractive for studies of various properties in wide-gap nitrides. The prerequisite condition for the success of any atomistic calculation is the availability of reliable interatomic potentials describing the interaction between atoms in the crystalline lattice. In contrast to the case for ionic materials such as oxides, semiconductors like silicon, and metals, there have been relatively few efforts devoted to the development of interatomic potentials for the group III nitrides. The only set of potentials of which we are aware were developed by Cormack [2] for AlN. No such efforts have been extended to the other members of the group III nitride family. In the present paper we derive a set of interatomic potentials for $\mathrm{GaN}$ and apply them to the study of the properties of $\mathrm{GaN}$. 


\section{The model}

In our atomistic description, the crystal is considered to consist of ions interacting via pair potentials and polarizable by means of the shell model [3]. GaN is often thought of as a covalent material and therefore it is questionable whether a description within the Born model is adequate. Here, we take guidance from first-principles calculations [4] which have assigned $+1.6 e$ and $-1.6 e$ charges to $\mathrm{Ga}$ and $\mathrm{N}$, respectively, using a Mulliken population analysis. This is strong evidence of a significant ionic character of GaN. Instead of the fully ionic charges $+3 e$ and $-3 e$, we choose the partially ionic model where charges of $+2 e$ and $-2 e$ are associated with $\mathrm{Ga}$ and $\mathrm{N}$, respectively, in the lattice. In general, covalent bonding requires an angle-dependent potential to describe its directional character. However, about a given point one can represent three-body effects as a sum of three two-body potentials. Unless there are some physical means of assigning terms independently, partitioning the energy between two- and three-body terms is not straightforward. It is suggested that one can either use a formal charged model with three-body angle bending terms or one can use a partially charged model with only two-body terms.

The two-body interatomic potential consists of a long-range Coulombic part and a shortrange part; the latter is represented here by an analytical expression of the Buckingham form for atoms $i$ and $j$ :

$$
V_{i j}=A \exp \left(-r_{i j} / \rho\right)-C r_{i j}^{-6}
$$

A cut-off radius of $10 \AA$ was taken for the short-range potentials. $\mathrm{N}$ ions are assumed to have massless shells, of charge $Y$, harmonically coupled to positive cores with a force constant $K$. The shell model reproduces the ion polarizability, $\alpha$; this quantity is related to the mechanical model by the following expression:

$$
\alpha=\frac{Y^{2}}{K} \text {. }
$$

The core and shell of the same ion do not interact Coulombically. The Ga ions are considered to be rigid ions.

The parameters of the short-range pair potentials, $A, \rho$ and $C$, as well as the nitrogen shell parameters, $Y$ and $K$, are obtained by fitting them to reproduce the properties of the wurtzite structure. These properties include lattice constants, and elastic and dielectric constants. The calculations were performed at $0 \mathrm{~K}$. However, because the potentials were fitted to experimental data they implicitly include thermal effects at the temperature of the experimental data measurement. We use both conventional fitting and relaxed fitting procedures [5] to achieve higher quality in our description of the crystal. In the relaxed fitting procedure the residual is defined by the errors in the structural parameters on optimization, rather than by using the forces in the experimental structure. While this is considerably more expensive, as it requires a complete optimization for each evaluation of the residual and finite-difference point for the derivatives of this quantity, it provides more accurate results as it allows for the fact that the displacements are related to the inverse Hessian matrix as well as the forces.

All of the calculations were performed using the program GULP [6] which utilizes efficient symmetry-adapted algorithms both for the evaluation of the energy and its derivatives, and also to accelerate the optimization procedure. In extreme cases this can lead to an order-of-magnitude reduction of the computer time. The electrostatic interactions are evaluated both in real and in reciprocal space according to the method of Ewald with the partitioning parameter, $\eta$, chosen so as to minimize the total number of terms to be 
evaluated in both series. Full details of the methodologies used by the program can be found elsewhere [6].

Table 1. Short-range potential and shell-model parameters for GaN. The charges on the ions are taken to be $-2 e$ and $2 e$ for anions and cations, respectively.

\begin{tabular}{lrlcll}
\hline & $A(\mathrm{eV})$ & $\rho(\AA)$ & $C\left(\mathrm{eV}^{6}\right)$ & $k\left(\mathrm{eV} \AA^{-9}\right)$ & $Y(\mathrm{e})$ \\
\hline $\mathrm{N}_{s}-\mathrm{N}_{s}$ & 4115.42 & 0.31949 & 280 & & \\
$\mathrm{Ga}_{c}-\mathrm{N}_{s}$ & 872.42 & 0.31318 & 0.0 & & \\
$\mathrm{Ga}_{c}-\mathrm{Ga}_{c}$ & 6068.14 & 0.31846 & 250 & & \\
$\mathrm{~N}_{c}-\mathrm{N}_{s}(\mathrm{GaN})$ & & & & 11.7092 & -2.5 \\
\hline
\end{tabular}

Table 2. Calculated and experimental bulk properties of GaN.

\begin{tabular}{|c|c|c|c|c|}
\hline \multirow[b]{2}{*}{ Property } & \multicolumn{2}{|c|}{ Wurtzite } & \multicolumn{2}{|c|}{ Zinc-blende } \\
\hline & Calculated & Experimental & Calculated & Experimental \\
\hline \multicolumn{5}{|c|}{ Structural parameter $(\AA)$} \\
\hline$a$ & 3.23 & $3.19^{\mathrm{a}}$ & 4.53 & 4.49 \\
\hline$c$ & 5.16 & $5.19^{\mathrm{a}}$ & & \\
\hline$u$ & 0.385 & $0.377^{\mathrm{b}}$ & & \\
\hline \multicolumn{5}{|c|}{ Elastic constant $\left(\right.$ dyn $\left.\mathrm{cm}^{-2}\right)$} \\
\hline$C_{11}$ & 38.63 & $39.1^{\mathrm{c}}$ & 29.96 & $29.6^{\mathrm{d}}$ \\
\hline$C_{12}$ & 15.98 & $14.3^{\mathrm{c}}$ & 19.07 & $15.4^{\mathrm{d}}$ \\
\hline$C_{13}$ & 14.13 & $10.8^{\mathrm{c}}$ & & \\
\hline$C_{33}$ & 39.12 & $39.2^{\mathrm{c}}$ & & \\
\hline$C_{44}$ & 11.50 & $10.3^{\mathrm{c}}$ & 15.95 & $20.6^{\mathrm{d}}$ \\
\hline$C_{66}$ & 11.32 & $12.4^{\mathrm{c}}$ & & \\
\hline \multicolumn{5}{|c|}{ Dielectric constant } \\
\hline$\epsilon_{0}^{11}$ & 8.05 & $9.38^{\mathrm{e}}$ & 8.88 & \\
\hline$\epsilon_{0}^{33}$ & 11.20 & $10.2^{\mathrm{e}}$ & & \\
\hline$\epsilon_{\infty}^{11}$ & 5.21 & $5.35^{\mathrm{e}}$ & 5.41 & \\
\hline$\epsilon_{\infty}^{33}$ & 5.84 & $5.35^{\mathrm{e}}$ & & \\
\hline
\end{tabular}

${ }^{\mathrm{a}}$ Xia et al [7].

${ }^{\mathrm{b}}$ Ueno et al [8].

${ }^{\mathrm{c}}$ Polian et al [9].

${ }^{\mathrm{d}}$ Calculated by Kim et al [10].

e Azuhata et al [22].

Table 1 lists the potential parameters representing the interatomic interactions in the lattice. The calculated lattice properties are compared with the experimental data in table 2 . The potential model reproduces the lattice structure well. For example, the calculated lattice constants are within $2 \%$ of the corresponding experimental values. The overall good agreement between the calculated and experimental properties for the wurtzite phase indicates the reliability of the interatomic potential set derived for GaN. 


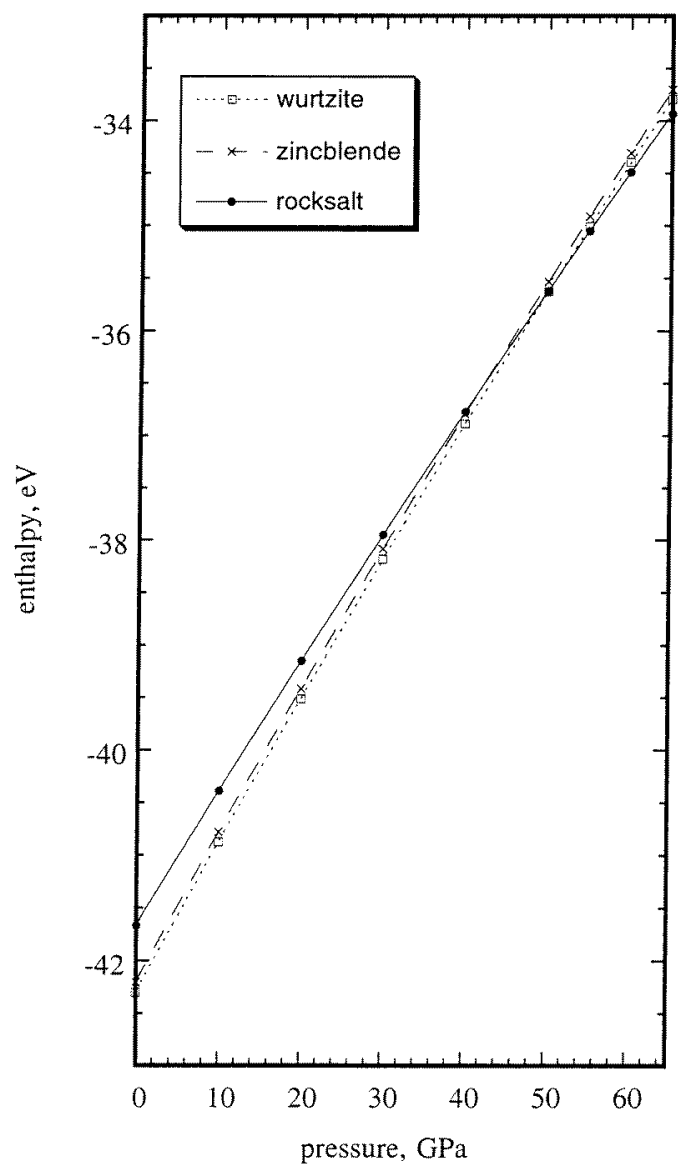

Figure 1. The calculated phase diagram of GaN.

\section{Results}

\subsection{Structure stability}

To find out which is the most stable structure under zero pressure we optimized the crystal structure parameters to yield the minimum total energy of the crystal for all three possible structures, namely hexagonal wurtzite, cubic zinc-blende and rock-salt structures. The total energy is equal to the internal energy of the crystal here, because both temperature and pressure are equal to zero. Note that the wurtzite structure is described by two lattice constants, $a$ and $c$, and the internal parameter $u$, whereas the zinc-blende and rock-salt structures are described by one lattice constant $(a)$ each. The calculated total energy per formula unit is lowest for the wurtzite phase, showing it to be the most stable phase. Hence, the derived potential set successfully predicts the structural stability of GaN under zero pressure. We also note here that the calculated lattice constant for the zinc-blende phase is $4.53 \AA$ which is in good agreement with the experimental value of $4.49 \AA$.

The bulk modulus can be derived from the elastic constants. We found it to be $228 \mathrm{GPa}$ for the wurtzite phase as compared to diverse experimental values of $188 \mathrm{GPa}$ [7], 
$237 \mathrm{GPa}$ [8] and $210 \mathrm{GPa}$ derived from experimental elastic constants [9]. There is no experimental determination of the bulk modulus for the zinc-blende phase available with which to compare our result, $227 \mathrm{GPa}$, but it is in reasonable agreement with the value of $201 \mathrm{GPa}$ obtained in density functional calculations [10]. Fitting their experimental data to a Birch first-order equation of state, Xia et al [7] gave an experimental bulk modulus of $248 \mathrm{GPa}$ for the rock-salt phase at zero pressure, as compared to the calculated value of $287 \mathrm{GPa}$.

\subsection{The phase diagram}

The applicability of the potential parameters to atomistic calculations is always questionable unless some evidence of their validity for a wide range of interatomic distances and coordination numbers is provided. For $\mathrm{GaN}$, an opportunity to test the reliability of the model exists due to its occurrence in different polytypes. At ambient conditions $\mathrm{GaN}$ crystallizes in the wurtzite phase although thin films of the zinc-blende polytype have been grown as well. At high pressure $(>35 \mathrm{GPa})$ it is found to undergo a transition to the rocksalt phase [7, 8]. Therefore, if one plots the phase enthalpy as a function of pressure, the wurtzite phase is expected to have the lowest enthalpy under low pressure and the rocksalt phase has the lowest enthalpy under pressure higher than the transition pressure. The zinc-blende phase is metastable and its enthalpy should never be the lowest one.

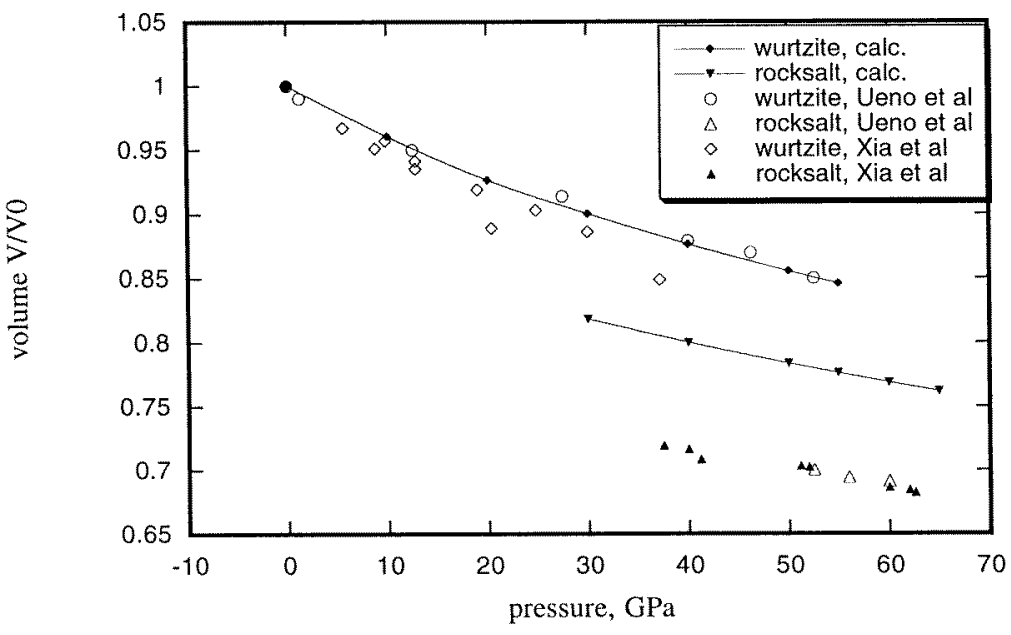

Figure 2. The equation of state for GaN. Calculated curves are compared to the experimental data $[7,8] . V_{0}$ is the unit-cell volume of the wurtzite phase at zero pressure.

Figure 1 shows the phase diagram calculated for all of the three phases considered here. Each structure was optimized to yield the minimum enthalpy at constant pressure to produce a point on the curve. The pressure was varied from 0 to $65 \mathrm{GPa}$ to obtain the phase diagram. As shown in figure 1, our model correctly reproduces the high-pressure phase transition from the wurtzite to the rock-salt phase occurring at $50 \mathrm{GPa}$. Experimental studies indicate a firstorder phase transition at about $37 \mathrm{GPa}$ [7] or $52 \mathrm{GPa}$ [8]. An equation of state is obtained from the same calculations as were used to derive the phase diagram, and it is shown in figure 2 together with experimental data. The calculated rock-salt structure lattice constant of $4.18 \AA$ differs from the experimental value of $4.01 \AA$ by less than $5 \%$. The difference 
is understandable, as it is clear that the effective radius of the nitride ion changes with the coordination number and therefore the best pair potentials for the two structures should be different. It is possible to achieve properly transferable potentials which will reproduce more than one coordination number accurately using a breathing shell model [11].

As expected, no phase transition is found to occur between the wurtzite and the zincblende phases because the zinc-blende phase enthalpy is always higher than that for the wurtzite phase. It is worth noting that whereas the mere existence of a transition has very little dependence on the potential parameters, we found that the transition pressure and volume contraction are sensitive to the dispersion term parameter, $C$. With an increase in this attractive interaction, the transition pressure decreases.

Our results show that the potential parameters obtained are suitable for describing both fourfold-coordinated and sixfold-coordinated structures. Note that both the number of nearest neighbours and the interatomic distances are significantly different in these two cases. Besides the fact that the model is able to successfully describe the phase transition, we gain more confidence as regards the applicability of our parameters to other situations when the coordination of the atoms changes, such as occurs in point defect calculations.

\subsection{Native defects}

As-grown GaN crystal has n-type conductivity due to the presence of intrinsic defects. The dominant donor has been identified as a nitrogen vacancy both experimentally [12] and theoretically $[13,14]$. The earliest theoretical estimates of defect formation enthalpy in $\mathrm{GaN}$ by Van Vechten [15] based on macroscopic considerations give the value of $3.9 \mathrm{eV}$ per defect for the Schottky defect. Recent LDA calculations [13, 14, 16] provide quantitative information on the energetics and geometry of the native defects in $\mathrm{GaN}$.

The formation energy of a defect is commonly defined as

$$
E_{f}=E_{t o t}-n_{\mathrm{Ga}} \mu_{\mathrm{Ga}}-n_{\mathrm{N}} \mu_{\mathrm{N}}-n_{\mathrm{e}} \mu_{\mathrm{e}}
$$

where $E_{t o t}$ is a directly calculated total energy of the crystal with defects, $n_{\mathrm{Ga}}$ and $n_{\mathrm{N}}$ are the numbers of respectively $\mathrm{Ga}$ and $\mathrm{N}$ atoms in the crystal, $n_{\mathrm{e}}$ is the number of electrons transferred to or from the defect, and the chemical potentials for $\mathrm{Ga}, \mathrm{N}$ atoms and the electrons are denoted by $\mu$ with the appropriate subscript. It is assumed in this model that vacancies and interstitials are created by neutral-atom exchange between the crystal and the corresponding solid or gaseous phases, and that atomic chemical potentials vary in the range restricted by the condition that their sum is equal to the chemical potential of bulk $\mathrm{GaN}$, thus maintaining equilibrium.

Our calculations of crystal disorder are restricted to calculations of Schottky and Frenkel defect formation energies. The Schottky defect formation energy is determined as the energy required to transfer infinitely separated bulk $\mathrm{Ga}$ and $\mathrm{N}$ ions to the crystal surface, whereas the Frenkel defect formation energy is the sum of the formation energies of infinitely separated vacancies and interstitials of the same kind of ion. Note that the formation energies of these defects do not involve atomic chemical potentials of the anion and cation separately, and therefore do not lead to differences between the Ga-rich and N-rich limits. We assumed that the charges of the vacancies and interstitials constituting these defects are determined by the charge of the bulk ion $(+2$ or -2 in our case). Thus, both Schottky and Frenkel defects are electrically neutral, which is equivalent to the condition $n_{\mathrm{e}}=0$, and their formation energy does not depend on the Fermi energy.

For defect calculations, GULP uses the Mott-Littleton method [17] where the crystal with a defect is divided into two regions. Atoms in the region immediately surrounding the 
defect are treated explicitly and they are relaxed to zero force. The outer region is treated approximately using macroscopic dielectric theory. In the present calculations the inner region consists of approximately 200 atoms, resulting in a total-energy accuracy better than $0.01 \mathrm{eV}$ with respect to the region size.

We found that defect formation energies for the zinc-blende phase do not greatly differ (less than $0.2 \mathrm{eV}$ for vacancies) from those in the wurtzite structure. Therefore, our calculated results refer to the zinc-blende phase only. Nitrogen and gallium interstitials were calculated for both tetrahedral and hexagonal positions, with the full relaxation of the surrounding lattice. It appeared that tetrahedral positions are more energetically favourable than hexagonal ones for both $\mathrm{N}$ (by $1.2 \mathrm{eV}$ ) and $\mathrm{Ga}$ (by $1.7 \mathrm{eV}$ ) interstitial ions. This is in agreement with previous results for $\mathrm{Ga}^{2+}$ interstitials in $\mathrm{GaAs}$ where tetrahedral positions were found to be the lowest-energy structures [18]. We note here that there are two inequivalent tetrahedral sites in the lattice in which the interstitial $\mathrm{Ga}$ is surrounded by either four $\mathrm{Ga}$ atoms $\left(\mathrm{T}_{\mathrm{d} 1}\right)$ or four $\mathrm{N}$ atoms $\left(\mathrm{T}_{\mathrm{d} 2}\right)$. Our calculations find the $\mathrm{T}_{\mathrm{d} 2}$ position for $\mathrm{Ga}^{2+}$ and the $\mathrm{T}_{\mathrm{d} 1}$ position for $\mathrm{N}^{2-}$ to have the lowest formation energy in the GaN lattice.

Our results indicate that the Schottky defect is dominant in GaN. The formation energies are 4.8, 6.1 and $6.9 \mathrm{eV}$ per defect for Schottky, Frenkel (N) and Frenkel (Ga) defects, respectively. They are similar to the corresponding values in binary oxides where the Schottky defect formation energy is typically about $3-5 \mathrm{eV}$ per defect. Formation energies of native defects in $\mathrm{GaN}$ as functions of the Fermi level energy were calculated by a first-principles method based on density functional theory $[14,16]$. For comparison with our atomistic results, we choose the values of the formation energies which correspond to a Fermi level of $2 \mathrm{eV}$ where the charge states of vacancies and interstitials are nearest to those used in the present atomistic study. These values are 3.3, 4.4 and $6.4 \mathrm{eV}$ for Schottky, Frenkel $(\mathrm{N})$ and Frenkel $(\mathrm{Ga})$ defects respectively. Note that the choice of the charge state strongly affects the energetics of the defect formation. Our results are in agreement with first-principles calculations $[14,16]$ in concluding that vacancies are the most probable defects in GaN.

The diffusion activation energy for a $\mathrm{N}$ vacancy is obtained as the difference in total energy between the fully optimized initial vacancy state and the lowest barrier state for a $\mathrm{N}$-ion movement from a neighbouring normal site into the vacancy. Three possible barrier positions for the nitrogen atom were considered. Here both tetrahedral and hexagonal interstitial positions and the point between two nitrogen vacancies were taken as starting points for the search for the barrier configuration for the migration of nitrogen atoms. After geometry optimization the latter position yields the lowest barrier height, $2.1 \mathrm{eV}$. The barrier geometry is determined by the displacement of the moving $\mathrm{N}$ ion from the initial position between two $\mathrm{N}$ sites by $0.49 \AA$ in the (100) direction. This atom is coordinated by one $\mathrm{Ga}$ ion and the distance between them is $1.84 \AA$. This position is similar to the (100) split-interstitial position which is one of the most stable positions for negatively charged As in GaAs. No experimental data are available for comparison with the calculated diffusion activation energy.

\subsection{Surface relaxation}

Recent first-principles calculations based on Hartree-Fock theory [19] and density functional theory $[19,20]$ have predicted an unusual surface relaxation of the nonpolar zinc-blende (110) surface. As compared to a variety of III-V compounds that exhibit a large surface bond rotation angle of about $29^{\circ}$, the much smaller surface $\mathrm{Ga}-\mathrm{N}$ bond rotation angle of about $9^{\circ}$ is expected [20] for the $\mathrm{GaN}(110)$ surface. The proposed relaxation mechanism involves 
redistribution of the electron density from the nitrogen dangling bond to the surface $\mathrm{Ga}-\mathrm{N}$ bond region, increasing the bond order. It is accompanied by the bond-length contraction by $5.2 \%$ of the bulk value.

Using the MARVIN [21] computer program package, which is designed to treat surfaces using the shell-model approach, we investigated the relaxation of the zinc-blende (110) surface. The top four layers of the surface constitute the region where pair interactions are explicitly calculated, whereas the rest of the semi-infinite crystal contributes only to the electrostatic potential. Coordinates of the atoms in the two topmost layers were optimized to yield the minimum total energy. The resulting equilibrium configuration is characterized by a surface layer rotation angle $\omega$ equal to $11.3^{\circ}$, corresponding to a $\mathrm{Ga}-\mathrm{N}$ bond rotation angle $\theta$ of $5.9^{\circ}$ and a surface bond contraction of $4.5 \%$ of the bulk bond length. As expected, the surface $\mathrm{Ga}$ ion moves downward and $\mathrm{N}$ moves upward from their ideal positions. It is worth noting that the shell of the surface nitrogen displaces towards the surface Ga ion, mimicking the electron density redistribution into the surface $\mathrm{Ga}-\mathrm{N}$ bond. It was found in ab initio calculations that surface back bonds also become stronger and shorten by $2 \%$. However, the sole shell of nitrogen in the shell-model calculations cannot reproduce the density redistribution in the back bonds. Accordingly, a small increase in the surface Ga (by $1.2 \%$ ) and $\mathrm{N}$ (by 3.7\%) back-bond lengths appeared as an artifact of the calculations. The prominent features of the unusual $\mathrm{GaN}(110)$ surface relaxation, namely surface bond-length contraction and small surface bond rotation angle, are therefore confirmed.

\section{Summary}

A set of interatomic potentials for $\mathrm{GaN}$ were developed in the framework of the shell-model approach. This model is able to successfully reproduce the structural, elastic and dielectric properties of the wurtzite, zinc-blende and rock-salt structures of the material. The highpressure phase transition properties were calculated and were shown to be in agreement with recent experimental data, thus verifying the general applicability of the model. Native-defect formation energies lead us to the conclusion that Schottky disorder will be the dominant type of disorder in undoped $\mathrm{GaN}$. The energy barrier for nitrogen vacancy diffusion was calculated to be $2.1 \mathrm{eV}$. The reliability of the model in treating low-coordination systems was confirmed in the study of the zinc-blende (110) surface relaxation. A surface bond contraction of about $5 \%$ and a layer rotation angle of $11^{\circ}$ are predicted. It is concluded that the interatomic potentials are suitable for the modelling of nitride properties over a wide range of interatomic distances and for varying coordination geometries of the ions.

\section{Acknowledgments}

The authors are indebted to Dr R Baetzold for his help in the calculations of surface relaxation. PZ and RP acknowledge financial support from AFOSR, Contract F49620-96-1-0319. JDG acknowledges the Royal Society for a University Research Fellowship.

\section{References}

[1] Nakamura S and Fasol G 1997 The Blue Laser Diode_GaN-based Light Emitters and Lasers (Berlin: Springer)

[2] Cormack A N 1989 J. Am. Ceram. Soc. 721730

[3] Dick B G and Overhauser A W 1958 Phys. Rev. 11290

[4] Pandey R, Jaffe J E and Harrison N M 1994 J. Phys. Chem. Solids 551357 
[5] Gale J D 1996 Phil. Mag. B 733

[6] Gale J D 1997 J. Chem. Soc. Faraday Trans. 93629

[7] Xia H, Xia Q and Ruoff A L 1993 Phys. Rev. B 4712925

[8] Ueno M, Yoshida M and Onodera A 1994 Phys. Rev. B 4914

[9] Polian A, Grimsditch M and Grzegorzy I 1996 J. Appl. Phys. 763343

[10] Kim K, Lambrecht W R L and Segall B 1996 Phys. Rev. B 5316310

[11] Wilson M, Madden P A, Pyper N C and Harding J H 1996 J. Chem. Phys. 1048068

[12] Perlin P, Suski T, Teisseyre H, Leszczynski M, Grzegorzy I, Jun J, Porowski S, Boguslawski P, Bernholc J, Chervin J C, Polian A and Moustakas T D 1995 Phys. Rev. Lett. 75296

[13] Boguslawski R, Briggs E, White T E, Wensell M G and Bernholc J 1994 Mater. Res. Soc. Symp. Proc. 339 693

[14] Neugebauer J and Van de Walle C G 1994 Mater. Res. Soc. Symp. Proc. 339687

[15] Van Vechten J A 1975 J. Electrochem. Soc. 122419

[16] Neugebauer J and Van de Walle C G 1994 Phys. Rev. B 508067

[17] Lidiard A B and Norgett M J 1972 Computational Solid State Physics ed F Herman et al (New York: Plenum) p 385

[18] Chadi D J 1992 Phys. Rev. B 469400

[19] Jaffe J E, Pandey R and Zapol P 1996 Phys. Rev. B 53 R4209

[20] Pandey R and Zapol P 1996 unpublished

[21] Gay D H and Rohl A C 1995 J. Chem. Soc. Faraday Trans. 91925

[22] Azuhata T, Sota T, Suzuki K and Nakamura S 1995 J. Phys.: Condens. Matter 7 L129 